\title{
Ueber eine Transformation der hydrodynamischen Gleichungen.
}

\author{
(Von Herrn H. Weber in Heidelberg.)
}

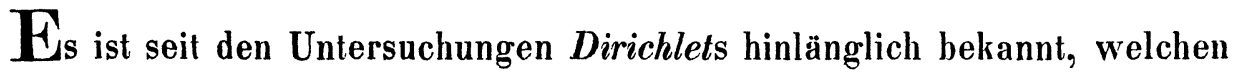
Vorzug die sogenannte Lagrangesche *) Form der Gleichungen der Hydrodynamik vor der Eulerschen Form voraus hat, dass nämlich das Gebiet der unabhängigen Variablen bei der ersteren ein für allemal gegeben ist, während in der zweiten Form dieses Gebiet wenigstens in den meisten Fällen erst von den zu suchenden unbekannten Grössen abhängt. Daher übersteigt die Behandlung der Eulerschen Gleichungen in allen den Fällen, wo die äussere Gestalt der flüssigen bewegten Masse nicht ungeändert bleibt, oder sich dieselbe nicht allerseits ins Unendliche erstreckt, bei weitem die Kräfte der heutigen Analysis, mehr als dies bei den Lagrangeschen Gleichungen trotz ihrer verwickelteren Form der Fall ist. Dem ungeachtet ist die Zahl der Probleme, die bis jetzt auf Grund der Lagrangeschen Gleichungen gelöst worden sind, eine ungemein geringe, und selbst die allgemeine Theorie dieser Gleichungen scheint mir noch nicht die Höhe erreicht zu haben, deren sie fähig ist. So ist namentlich die Frage noch nicht beantwortet, welchen Nutzen man für die Integration der Lagrangeschen Gleichungen ziehen kann aus der Annahme eines Geschwindigkeitspotentials, eine Annahme, wodurch die Eulerschen Gleichungen so ausserordentlich vereinfacht werden. Da die Voraussetzung des Geschwindigkeitspotentials nicht bloss eine analytische, sondern eine physikalische Vereinfachung des Problems ist, so muss auch in der Lagrangeschen Form der Gleichungen durch dieselbe eine Erleichterung herbeigeführt werden.

Ich werde im Folgenden eine Form der hydrodynamischen Gleichungen entwickeln, in der ihnen der wesentliche Vortheil der Eulerschen Gleichungen zukommt, nämlich dass die darin vorkommenden Differentialquotienten nur von der ersten Ordnung sind, und dass die Bedingung der Existenz eines Geschwindigkeitspotentials sofort eingeführt werden kann, und zwar ohne die Zahl der

*) Ich bediene mich der gewöhnlichen Bezeichnung wiewoll es mir nicht unbekannt ist, dass, wie Herr Hankel bemerkt hat, beide Formen der hydrodynamischen Gleichungen von Euler herrühren. 
unbekannten Functionen und der Differentialgleichungen zu vermehren. Diese Gleichungen erster Ordnung haben zwar nicht die Einfachheit der Eulerschen Gleichungen, besitzen aber gleichzeitig den Vortheil der Lagrangeschen Gleichungen, nämlich dass das Gebiet der unabhängigen Veränderlichen unveränderlich und gegeben ist.

\section{\$. 1.}

Ich gehe von der bekannten Form der Lagrangeschen Gleichungen aus. Bezeichnen $x y z$ die rechtwinkligen Coordinaten eines Flüssigkeitstheilchens, dessen Coordinaten beim Beginn der Bewegung $a b c$ sind; bezeichnet ferner $p$ den Druck, $\varrho$ die Dichtigkeit, $V$ das Potential der wirkenden Kräfte, alles bezogen auf das Theilchen $a b c$ zur Zeit $t$, so sind diese Gleichungen folgende:

$$
\left\{\begin{array}{l}
\frac{\partial^{2} x}{\partial t^{2}} \frac{\partial x}{\partial a}+\frac{\partial^{2} y}{\partial t^{2}} \frac{\partial y}{\partial a}+\frac{\partial^{2} z}{\partial t^{2}} \frac{\partial z}{\partial a}-\frac{\partial V}{\partial a}+\frac{1}{\rho} \frac{\partial p}{\partial a}=0 \\
\frac{\partial^{2} x}{\partial t^{2}} \frac{\partial x}{\partial b}+\frac{\partial^{2} y}{\partial t^{2}} \frac{\partial y}{\partial b}+\frac{\partial^{2} z}{\partial t^{2}} \frac{\partial z}{\partial b}-\frac{\partial V}{\partial b}+\frac{1}{\rho} \frac{\partial p}{\partial b}=0 \\
\frac{\partial^{2} x}{\partial t^{2}} \frac{\partial x}{\partial c}+\frac{\partial^{2} y}{\partial t^{2}} \frac{\partial y}{\partial c}+\frac{\partial^{2} z}{\partial t^{2}} \frac{\partial z}{\partial c}-\frac{\partial V}{\partial c}+\frac{1}{\rho} \frac{\partial p}{\partial c}=0 .
\end{array}\right.
$$

Dazu kommt für incompressible Flüssigkeiten die Gleichung

$$
\text { (2.) } \quad \Sigma \pm \frac{\partial x}{\partial a} \frac{\partial y}{\partial b} \frac{\partial z}{\partial c}=1
$$

und für Gase:

$$
\left(\boldsymbol{2}^{\prime} .\right) \quad \Sigma \pm \frac{\partial x}{\partial a} \frac{\partial y}{\partial b} \frac{\partial z}{\partial c}=\frac{\varrho_{0}}{\varrho},
$$

wo $\varrho_{0}$ die Dichtigkeit an der Stelle $a b c$ beim Anfang der Bewegung bedeutet. Für incompressible Flüssigkeiten ist $\varrho$ eine Constante, die $=1$ gesetzt werden kann, während bei Gasen $\rho$ als eine erfahrungsmässig bekannte Function von $p$ zu betrachten ist, so dass die Anzahl der Gleichungen der Anzahl der zu bestimmenden Functionen in beiden Fällen gleich kommt.

Ich schicke einige Betrachtungen über die Grenz- und Stetigkeitsbedingungen voraus, welche erforderlich sind, um die unbekannten Functionen vollständig zu bestimmen.

Die Ableitung der Gleichung (2.) und ( $2^{\prime}$.) setzt bekanntlich voraus, dass diejenigen Flüssigkeitstheilchen, welche $\mathrm{zu}$ irgend einer Zeit auf einer geschlossenen Fläche liegen, auch zu jeder anderen Zeit eine geschlossene Fläche bilden, und dass die Theilchen, welche innerhalb einer solchen Fläche 
liegen, auch beständig innerhalb derselben bleiben, woraus unmittelbar folgt, dass die Oberfläche der ganzen bewegten Flüssigkeit beständig von denselben Theilchen gebildet wird. Diese Bedingung lässt sich analytisch so ausdrücken, dass $x y z \mathrm{zu}$ allen Zeiten einwerthige, stetige und für endliche Werthe der Variablen endliche Functionen von $a b c$ sein müssen, innerhalb des diesen Variablen zukommenden Bereichs, und dass den Grössen $a b c$ als Functionen

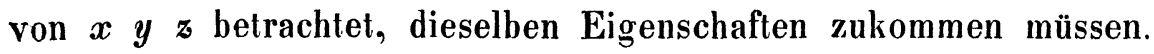

Die letzteren dieser Bedingungen sind aber wegen der Gleichungen (2.) oder $\left(\boldsymbol{2}^{\prime}\right.$.) Folgen der ersteren, wenn man noch die Bedingung hinzufügt, dass auch die Geschwindigkeiten $\frac{\partial x}{\partial t}, \frac{\partial y}{\partial t}, \frac{\partial z}{\partial t}$ für alle Zeiten endliche und stetige Functionen von $a b$ sein sollen.

Betrachtet man nämlich irgend zwei Functionensysteme: $x y z, x^{\prime} y^{\prime} z^{\prime}$, welche sich auf zwei verschiedene Werthe von $t$ beziehen, und beide endliche und stetige Functionen von $a b c$ sind, so müssen auch die $x^{\prime} y^{\prime} z^{\prime}$ endliche und stetige Functionen der $x y z$ sein und umgekehrt, mithin auch $a b c$ endliche und stetige Functionen von $x y z$. Denn bildet man die Differentialquotienten $\frac{\partial x^{\prime}}{\partial x} \cdots$ aus den Gleichungen:

$$
\frac{\partial x^{\prime}}{\partial \boldsymbol{a}}=\frac{\partial x^{\prime}}{\partial x} \frac{\partial x}{\partial \boldsymbol{a}}+\frac{\partial x^{\prime}}{\partial y} \frac{\partial y}{\partial a}+\frac{\partial x^{\prime}}{\partial z} \frac{\partial z}{\partial \boldsymbol{a}}
$$

so erhalten die Auflösungen den Nenner $\Sigma \pm \frac{\partial x}{\partial a} \frac{\partial y}{\partial b} \frac{\partial z}{\partial c}$, welcher jedenfalls nicht verschwinden kann, da auch bei Gasen die Dichtigkeit nicht unendlich und nicht Null werden kann. Die Differentialquotienten $\frac{\partial x^{\prime}}{\partial x}, \frac{\partial x^{\prime}}{\partial y} \cdots$ sind sonach alle endlich und mithin die Functionen $x^{\prime} y^{\prime} z^{\prime}$ in Bezug auf $x y z$ endlich und stetig.

Um den Nachweis zu führen, dass unter der Voraussetzung, dass $x y z$ zu allen Zeiten einwerthige Functionen von $a b c$ sind, auch nicht zwei Theilchen, welche zu einer Zeit verschiedene Coordinaten haben, zu einer anderen Zeit dieselben Coordinaten haben können, betrachten wir zwei Flüssigkeitstheilchen, welche zur Zeit $t$ respective die Coordinaten $x y z, x^{\prime} y^{\prime} z^{\prime}$ haben mögen, und setzen voraus, dass $x$ von $x^{\prime}, y$ von $y^{\prime}, z$ von $z^{\prime}$ nur unendlich wenig verschieden sei. Wenn nun diese beiden Theilchen nach Verlauf des Zeitelements $d t$ dieselben Coordinaten hätten, so müsste sein: 


$$
\begin{aligned}
& \left(x-x^{\prime}\right)+\frac{d\left(x-x^{\prime}\right)}{d t} d t=0, \\
& \left(y-y^{\prime}\right)+\frac{d\left(y-y^{\prime}\right)}{d t} d t=0, \\
& \left(z-z^{\prime}\right)+\frac{d\left(z-z^{\prime}\right)}{d t} d t=0 .
\end{aligned}
$$

Wegen der vorausgesetzten Stetigkeit können die Theilchen $x y z, x^{\prime} y^{\prime} z^{\prime}$ auch beim Beginn der Bewegung nur unendlich wenig von einander entfernt gewesen sein. Bezeichnet man also die Unterschiede der Anfangswerthe der Coordinaten mit $d a, d b, d c$, so lässt sich immer eine unendlich kleine Grösse $\varepsilon$ der Art bestimmen, dass

$$
d a=\varepsilon \alpha, \quad d b=\varepsilon \beta, \quad d c=\varepsilon \gamma
$$

und dass die Grössen $\alpha \beta \gamma$ jedenfalls nicht alle drei unendlich klein sind. Dann lassen sich die obigen Gleichungen so schreiben:

$$
\begin{aligned}
& \frac{\partial x}{\partial a} \alpha+\frac{\partial x}{\partial b} \beta+\frac{\partial x}{\partial c} \gamma=-\left\{\frac{\partial^{2} x}{\partial a \partial t} \alpha+\frac{\partial^{2} x}{\partial b \partial t} \beta+\frac{\partial^{2} x}{\partial c \partial t} \gamma\right\} d t=\varepsilon_{1}, \\
& \frac{\partial y}{\partial a} \alpha+\frac{\partial y}{\partial b} \beta+\frac{\partial y}{\partial c} \gamma=-\left\{\frac{\partial^{2} y}{\partial a \partial t} \alpha+\frac{\partial^{2} y}{\partial b \partial t} \beta+\frac{\partial^{2} y}{\partial c \partial t} \gamma\right\} d t=\varepsilon_{2}, \\
& \frac{\partial z}{\partial a} \alpha+\frac{\partial z}{\partial b} \beta+\frac{\partial z}{\partial c} \gamma=-\left\{\frac{\partial^{2} z}{\partial a \partial t} \alpha+\frac{\partial^{2} z}{\partial b \partial t} \beta+\frac{\partial^{2} z}{\partial c \partial t} \gamma\right\} d t=\varepsilon_{3} .
\end{aligned}
$$

Sind nun die Functionen $\frac{\partial x}{\partial t}, \frac{\partial y}{\partial t}, \frac{\partial z}{\partial t}$ zu allen Zeiten stetige und endliche Functionen von $a b c$, so sind die $\varepsilon_{1} \varepsilon_{2} \varepsilon_{3}$ unendlich kleine Grössen von der Ordnung $d t$, und wenn man die vorstehenden Gleichungen in Bezug auf die auf der linken Seite stehenden $\alpha \beta \gamma$ auflöst, so ergeben sich diese, da die Determinante nicht verschwinden kann, gleichfalls als unendlich kleine Grössen von der Ordnung $d t$, was gegen die Voraussetzung ist.

Wegen der Stetigkeit können auch nicht Theilchen zusammenfallen, welche unmittelbar vorher durch eine endliche Strecke getrennt waren. Demnach reduciren sich die Stetigkeitsbedingungen auf folgende: die Coordinaten $x y z$ und die Geschwindigkeiten $\frac{d x}{d t}, \frac{d y}{d t}, \frac{d z}{d t}$ müssen für alle Werthe von $t$ einwerthige, stetige und für endliche Werthe der Variablen endliche Functionen von $\begin{array}{llll}a & b & c & \text { sein. }\end{array}$

Dazu tritt noch die eine Bedingung, dass entweder der Druck zu allen Zeiten eine stetige Function des Ortes ist, oder dass die Geschwindigkeiten stetige Functionen der Zeit sind; von diesen beiden letzteren Bedingungen ist 
wegen der Gleichungen (1.) unter der Voraussetzung stetiger Kräfte die eine eine Folge der andern.

Dazu kommen noch die Bedingungen des Anfangszustandes und der Grenzen. Die ersteren sind einfach die, dass zur Zeit $t=0, x=a, y=b$, $z=c$ sein muss, und dass die Geschwindigkeiten $\frac{d x}{d t}, \frac{d y}{d t}, \frac{d z}{d t}$ für $t=0$ beliebig gegebene Functionen $u_{0}, v_{0}, w_{0}$ von $a b c$ werden, welche bei incompressiblen Flüssigkeiten der Bedingung genügen müssen:

$$
\frac{\partial u_{0}}{\partial a}+\frac{\partial v_{0}}{\partial b}+\frac{\partial w_{0}}{\partial c}=0 \text {. }
$$

Die Bedingungen, die während der ganzen Dauer der Bewegung an der Grenze erfüllt sein müssen, sind je nach der speciellen Natur des Problems sehr verschieden. Ist z. B. die ganze Oberfläche einer incompressiblen Flüssigkeit frei beweglich, so ist weiter nichts erforderlich, als dass an der ganzen Oberfläche der Druck gegeben ist. Ist ein Theil der Oberfläche von festen oder bewegten Wänden gebildet, so müssen, der gewöhnlichen Annahme zufolge, die Theilchen, welche zu Anfang der Wand angehörten, während der ganzen Bewegung mit der Wand in Berührung bleiben, während an dem freien Theil der Oberfläche wieder der Druck gegeben sein muss. Aus physikalischen Gründen ist anzunehmen, dass durch diese Bedingungen die Bewegung eine vollständig gegebene ist. Der Nachweis aber, dass auch mathematisch durch diese Bedingungen das Problem der Integration der Gleichungen ein völlig eindeutiges ist, dürfte ziemlich schwierig sein.

\$. 2.

Um nun die oben erwähnte Transformation der hydrodynamischen Gleichungen auf Differentialgleichungen der ersten Ordnung durchzuführen, multiplicirt man die Gleichungen (1.) mit $d t$ und integrirt dieselben zwischen den Grenzen 0 und $t$. Wendet man dann auf die drei ersten Glieder jeder dieser Gleichungen eine theilweise Integration an, welche wegen der vorausgesetzten Stetigkeitsbedingungen statthaft ist, und beachtet man, dass man für die untere Grenze $t=0$ hat:

$$
\begin{array}{llll}
\frac{\partial x}{\partial a}=1, & \frac{\partial x}{\partial b}=0, & \frac{\partial x}{\partial c}=0 \\
\frac{\partial y}{\partial a}=0, & \frac{\partial y}{\partial b}=1, & \frac{\partial y}{\partial c}=0 \\
\frac{\partial z}{\partial a}=0, & \frac{\partial z}{\partial b}=0, & \frac{\partial z}{\partial c}=1 \\
\frac{\partial x}{\partial t}=u_{0}, & \frac{\partial y}{\partial t}=v_{0}, & \frac{\partial z}{\partial t}=w_{0}
\end{array}
$$


so erhält man ohne Schwierigkeiten:

$$
\left\{\begin{array}{l}
\frac{\partial x}{\partial t} \frac{\partial x}{\partial a}+\frac{\partial y}{\partial t} \frac{\partial y}{\partial a}+\frac{\partial z}{\partial t} \frac{\partial z}{\partial a}-u_{0}=\frac{\partial \lambda}{\partial a} \\
\frac{\partial x}{\partial t} \frac{\partial x}{\partial b}+\frac{\partial y}{\partial t} \frac{\partial y}{\partial b}+\frac{\partial z}{\partial t} \frac{\partial z}{\partial b}-v_{0}=\frac{\partial \lambda}{\partial b} \\
\frac{\partial x}{\partial t} \frac{\partial x}{\partial c}+\frac{\partial y}{\partial t} \frac{\partial y}{\partial c}+\frac{\partial z}{\partial t} \frac{\partial z}{\partial c}-w_{0}=\frac{\partial \lambda}{\partial c}
\end{array},\right.
$$

worin zur Abkürzung gesetzt ist:

$$
\lambda=\int_{0}^{t}\left\{V-\int \frac{\partial p}{\rho}+\frac{1}{2}\left(\left(\frac{\partial x}{\partial t}\right)^{2}+\left(\frac{\partial y}{\partial t}\right)^{2}+\left(\frac{\partial z}{\partial t}\right)^{2}\right)\right\} d t .
$$

Wenn man nun $\lambda$ als eine neue abhängige Variable ansieht, so bilden die Gleichungen (3.) ein System partieller Differentialgleichungen erster Ordnung, welche in Verbindung mit der Gleichung (2.) oder $\left(\boldsymbol{z}^{\prime}\right)$ und mit der neuen Gleichung

$$
\text { (4.) } \frac{\partial \lambda}{\partial t}=V-\int \frac{\partial p}{\rho}+\frac{1}{2}\left\{\left(\frac{\partial x}{\partial t}\right)^{2}+\left(\frac{\partial y}{\partial t}\right)^{2}+\left(\frac{\partial z}{\partial t}\right)^{2}\right\}
$$

der Zahl nach genügen, um die fünf Functionen: $x y z \lambda p$ zu bestimmen, da $\int \frac{d p}{\varrho}$ als eine durch die Erfahrung gegebene Function von $p$ zu betrachten ist, welche im Fall einer incompressiblen Flüssigkeit, wo constant ist, in $\frac{p}{\rho}$ übergeht, so dass, wenn man $\varrho=1$ setzt, die Gleichung (4.) für diesen Fall wird:

$$
\text { (4.') } \quad \frac{\partial \lambda}{\partial t}=V-p+\frac{1}{2}\left\{\left(\frac{\partial x}{\partial t}\right)^{2}+\left(\frac{\partial y}{\partial t}\right)^{2}+\left(\frac{\partial z}{\partial t}\right)^{2}\right\} \text {. }
$$

Die Function $\lambda$ muss die Bedingung des Anfangszustandes erfüllen, dass für $t=0, \lambda=0$ werde, was unmittelbar aus der Definition von $\lambda$ hervorgeht, während die Bedingungen des Anfangszustandes für die Geschwindigkeiten $\frac{\partial x}{\partial t} \frac{\partial y}{\partial t} \frac{\partial z}{\partial t}$ von selbst erfüllt sind, wenn die Functionen $x y z \lambda p$ die Gleichungen (3.), (4.) und die übrigen Bedingungen des Anfangszustandes befriedigen. Die Gleichungen (3.), (4.) sind vollständig äquivalent mit den Gleichungen (1.), denn wenn man aus den ersteren $\lambda$ durch Differentiation eliminirt, so gelangt man direct zu den Gleichungen (1.) zurück.

\section{\$. 3.}

Durch diese Transformation sind nun die hydrodynamischen Gleichungen in der That auf Gleichungen der ersten Ordnung zurückgeführt. Allerdings ist dabei scheinbar die Zahl der unbekannten Functionen und der Differential- 
gleichungen um eins vermehrt. Beachtet man aber, dass bei den incompressiblen Flüssigkeiten die Function $p$ in den Gleichungen (3.), (2.) gar nicht vorkommt, und dass selbst bei Gasen diese Function mittelst der Gleichung (4.) aus ( $2^{\prime}$.) eliminirt werden kann, so erkennt man, dass die Gleichungen (3.), (2.) oder ( $2^{\prime}$.) für sich integrirt werden können, und dass dann nachträglich die Function $p$ aus der Gleichung (4.) bestimmt wird. In den Fällen, wo die Function $p$ an einem Theil der Oberfläche oder an der ganzen Oberfläche gegeben ist, kann die Gleichung (4.) oder (4'.) unmittelbar auf den Theil der Grenze angewandt werden, wo $p$ gegeben ist, und enthält dann nur noch die unbekannten Functionen $x y z$ und $\lambda$. Sie vertritt in diesen Fällen die Stelle einer Grenzbedingung für diese Functionen und dient zur vollständigen Bestimmung derselben. Es ist also in der That, was die Integration anlangt, die Anzahl der unbekannten Functionen nicht vermehrt worden.

In dieser Form der hydrodynamischen Gleichungen lässt sich nun leicht auch die Voraussetzung eines Geschwindigkeitspotentials einführen.

Man hat nur anzunehmen, dass die Functionen $u_{0} v_{1} w_{0}$ die partiellen Differentialquotienten einer Function $\mu$ nach $a b c$ sind.

Man kann dann in den Gleichungen (3.) die Function $\mu$ mit $\lambda$ vereinigen, wodurch dieselben die Gestalt annehmen:

$$
\begin{aligned}
& \frac{\partial x}{\partial t} \frac{\partial x}{\partial a}+\frac{\partial y}{\partial t} \frac{\partial y}{\partial a}+\frac{\partial z}{\partial t} \frac{\partial z}{\partial \boldsymbol{a}}=\frac{\partial \lambda}{\partial \boldsymbol{a}} \\
& \frac{\partial \boldsymbol{x}}{\partial t} \frac{\partial \boldsymbol{x}}{\partial \boldsymbol{b}}+\frac{\partial y}{\partial t} \frac{\partial y}{\partial b}+\frac{\partial z}{\partial t} \frac{\partial z}{\partial b}=\frac{\partial \lambda}{\partial \boldsymbol{b}} \\
& \frac{\partial x}{\partial t} \frac{\partial x}{\partial \boldsymbol{c}}+\frac{\partial y}{\partial t} \frac{\partial y}{\partial c}+\frac{\partial z}{\partial t} \frac{\partial z}{\partial \boldsymbol{c}}=\frac{\partial \lambda}{\partial \boldsymbol{c}}
\end{aligned}
$$

Die Function $\lambda$ ist dann so zu bestimmen, dass sie zur Zeit $t=0$ nicht verschwindet, sondern $\mu$ wird. Dadurch sind also die Functionen $u_{0} v_{0} w_{0}$ aus den Differentialgleichungen entfernt und in die Bedingungen des Anfangszustandes verlegt, wodurch eine wesentliche Erleichterung des Problems der Integration der partiellen Differentialgleichungen herbeigeführt wird.

Heidelberg, im October 1867. 